\title{
Realistic Reference for Evaluation of Vehicle Safety Focusing on Pedestrian Head Protection Observed From Kinematic Reconstruction of Real-World Collisions
}

\author{
Guibing $\mathrm{Li}^{1}$, Jinming $\mathrm{Liu}^{1}$, Kui $\mathrm{Li}^{2 *}$, Hui Zhao ${ }^{2 *}$, Liangliang Shi ${ }^{3}$, Shuai Zhang ${ }^{4}$ and Jin $\mathrm{Nie}^{5}$ \\ ${ }^{1}$ School of Mechanical Engineering, Hunan University of Science and Technology, Xiangtan, China, ${ }^{2}$ Chongqing Key Laboratory \\ of Vehicle Crash/Bio-Impact and Traffic Safety, Institute for Traffic Medicine, Daping Hospital, Army Medical University, \\ Chongqing, China, ${ }^{3}$ China Automotive Engineering Research Institute Co., Ltd., Chongqing, China, ${ }^{4}$ The Fifth Institute of Army \\ Academy, Wuxi, China, ${ }^{5}$ Loudi Vocational and Technical College, Loudi, China
}

OPEN ACCESS

Edited by:

David W. Wright,

Emory University, United States

Reviewed by:

Richie Gill,

University of Bath, United Kingdom

Jonathan Rupp,

Emory University, United States

*Correspondence:

Kui Li

likui0708@126.com

Hui Zhao

box.zhaohui@163.com

Specialty section: This article was submitted to Biomechanics,

a section of the journal Frontiers in Bioengineering and Biotechnology

Received: 01 September 2021 Accepted: 22 November 2021 Published: 21 December 2021

Citation:

Li G, Liu J, LiK, Zhao H, Shi L, Zhang S and Nie J (2021) Realistic Reference for Evaluation of Vehicle Safety Focusing on Pedestrian Head Protection Observed From Kinematic Reconstruction of RealWorld Collisions.

Front. Bioeng. Biotechnol. 9:768994. doi: 10.3389/fbioe.2021.768994
Head-to-vehicle contact boundary condition and criteria and corresponding thresholds of head injuries are crucial in evaluation of vehicle safety performance for pedestrian protection, which need a constantly updated understanding of pedestrian head kinematic response and injury risk in real-world collisions. Thus, the purpose of the current study is to investigate the characteristics of pedestrian head-to-vehicle contact boundary condition and pedestrian AIS3+ (Abbreviated Injury Scale) head injury risk as functions of kinematic-based criteria, including HIC (Head Injury Criterion), HIP (Head Impact Power), GAMBIT (Generalized Acceleration Model for Brain Injury Threshold), RIC (Rotational Injury Criterion), and BrIC (Brain Injury Criteria), in real-world collisions. To achieve this, 57 vehicle-to-pedestrian collision cases were employed, and a multi-body modeling approach was applied to reconstruct pedestrian kinematics in these real-world collisions. The results show that head-to-windscreen contacts are dominant in pedestrian collisions of the analysis sample and that head WAD (Wrap Around Distance) floats from 1.5 to $2.3 \mathrm{~m}$, with a mean value of $1.84 \mathrm{~m} ; 80 \%$ of cases have a head linear contact velocity below $45 \mathrm{~km} / \mathrm{h}$ or an angular contact velocity less than $40 \mathrm{rad} / \mathrm{s}$; pedestrian head linear contact velocity is on average $83 \pm 23 \%$ of the vehicle impact velocity, while the head angular contact velocity (in $\mathrm{rad} / \mathrm{s}$ ) is on average $75 \pm 25 \%$ of the vehicle impact velocity in $\mathrm{km} / \mathrm{h} ; 77 \%$ of cases have a head contact time in the range 50-140 ms, and negative and positive linear correlations are observed for the relationships between pedestrian head contact time and WAD/height ratio and vehicle impact velocity, respectively; $70 \%$ of cases have a head contact angle floating from $40^{\circ}$ to $70^{\circ}$, with an average value of $53^{\circ}$; the pedestrian head contact angles on windscreens (average $=48^{\circ}$ ) are significantly lower than those on bonnets (average $=60^{\circ}$ ); the predicted thresholds of $\mathrm{HIC}$, HIP, GAMBIT, RIC, BrIC2011, and BrIC2013 for a 50\% probability of AIS3+ head injury risk are $1,300,60 \mathrm{~kW}, 0.74,1,470 \times 10^{4}, 0.56$, and 0.57 , respectively. The findings of the current work could provide realistic reference for evaluation of vehicle safety performance focusing on pedestrian protection.

Keywords: pedestrian head-to-vehicle contact, boundary condition, injury risk, kinematic reconstruction, real-world accidents 


\section{BACKGROUND}

In vehicle-to-pedestrian crashes, injuries to the head account for more than $30 \%$ of all AIS2+ (Abbreviated Injury Scale) injuries to pedestrians (Mizuno, 2005), which are also the main causes of pedestrian death (Yang, 2005; Simms and Wood, 2009; Schmitt et al., 2010). Hence, pedestrian head protection is one of the most important assessments of vehicle safety performance in regulations, such as the New Car Assessment Programs (NCAPs) in different countries and regions (C-NCAP, 2020; Euro-NCAP, 2020; J-NCAP, 2014), which have significantly improved current vehicle safety design (Li et al., 2018 and 2019). In NCAPs, subsystem tests using isolated impactors with the consideration of head-to-bonnet/windshield area impacts are employed, where the definition of the impact boundary condition and the criterion and corresponding thresholds for head injuries are crucial since these have a significant influence on vehicle safety (pedestrian injury risk) rating (C-NCAP, 2020; Euro-NCAP, 2020). Thus, a good understanding of pedestrian head-to-vehicle contact boundary condition and head injury risk in real-world collisions can help improve the effectiveness of head impactor subsystem tests in vehicle safety evaluation.

In subsystem tests, the head-to-vehicle contact boundary condition is defined as a constant linear impact speed at a certain angle (relative to the horizontal plane) to the head impactor; e.g., the C-NCAP and C-IASI use a linear head impact speed of $40 \mathrm{~km} / \mathrm{h}$ at $65^{\circ}$ for adult head impactor tests (C-IASI, 2017; C-NCAP, 2020), which is the same as defined in the Euro-NCAP (2020). However, the head-to-vehicle contact boundary condition defined in the current C-NCAP and C-IASI may not suit the situation in China due to the differences in characteristics of traffic accidents between China and Europe as reported in previous studies (Chen et al., 2009; Yang and Otte, 2007). Moreover, the rotational kinematics of pedestrian head-tovehicle contact is not considered in the current NCAPs, which is an important factor affecting head injury risk (Gennarelli et al., 1971; Rowson et al., 2011; Takhounts et al., 2011 and 2013). On the other hand, NCAPs in different countries and regions currently use the Head Injury Criterion (HIC) and relative thresholds to assess pedestrian head injury risk (C-NCAP, 2020; Euro NCAP, 2020). However, the HIC is controversial for predicting brain injury risk as it only considers head linear acceleration while some brain injuries are usually caused by rotational motion or combining linear motion (Schmitt et al., 2010; Simms and Wood, 2009; Yang, 2005). Given this, some other kinematic-based criteria were proposed, considering rotational response only or combining linear and rotational response for Traumatic Brain Injury (TBI), such as the Head Impact Power (HIP) (Newman et al., 2000), Generalized Acceleration Model for Brain Injury Threshold (GAMBIT) (Newman, 1986), Rotational Injury Criterion (RIC) (Kimpara and Iwamoto, 2012), and Brain Injury Criteria (BrIC) (Takhounts et al., 2011 and 2013). Accordingly, the thresholds or risk curves of human head injuries at different AIS levels were also proposed for these criteria, based on data from physical impact tests, reconstruction of real-world accidents (football players, motorcyclists, pedestrians, etc.) using isolated Anthropomorphic Test Devices (ATDs) and/or numerical human body models of head (Kimpara and Iwamoto, 2012; Marjoux et al., 2008; Newman, 1986; Takhounts et al., 2011 and 2013). However, few studies have focused on developing injury risk curves from reconstruction of real-world pedestrian crashes at the full-body level, covering all the above-mentioned kinematic-based criteria, where the influence of restriction from the torso and lower body on pedestrian head dynamic response and the cumulative head kinematics in the whole impact can be considered.

Therefore, the purpose of the current study is to investigate the characteristics of pedestrian head-to-vehicle contact boundary condition and to develop pedestrian head injury risk curves via kinematic reconstruction of real-world collisions. To achieve this, 57 vehicle-to-pedestrian collision cases were employed, and a multi-body modeling approach was applied to reconstruct pedestrian kinematics in these real-world collisions. Based on the reconstruction data, the characteristics of pedestrian head-tovehicle contact boundary condition were then analyzed, and the pedestrian head injury risk curves as functions of kinematicbased criteria (HIC, HIP, GAMBIT, RIC, and BrIC) were built. The findings of the current work can provide realistic reference for evaluation of vehicle safety focusing on pedestrian head protection.

\section{METHODS}

\section{Collision Data}

In the current work, 57 vehicle-to-pedestrian collision cases were selected, where 38 cases with video data captured during 2012-2019 were from the database of the Institute for Traffic Medicine, Army Military University, China, and another 19 cases were from the database of IVAC (In-Depth Investigation of Vehicle Accidents in Changsha). These enrolled cases have met the following inclusion criteria: (1) at least one AIS1+ head injury was recorded using the AIS 2005 classification (Gennarelli and Wodzin, 2006), (2) collisions involved passenger cars, (3) pedestrians are adult and were hit only once by a vehicle's front, (4) information on vehicle (model and year), pedestrian (gender, height, weight, and age), and injuries was recorded, and (5) the collision information (vehicle impact speed, initial contact location, etc.) can be calculated/estimated. Supplementary Appendix Table S1 shows the general information of these 57 cases, where the average height for female is $157 \mathrm{~cm}$ and that for male is $170 \mathrm{~cm}$, which generally match the stature of the Chinese population. In this sample, 39 cases are sedan crashes, while the other 18 cases are collisions with SUV (Sport Utility Vehicle) or MPV (Multi-purpose Vehicles).

\section{Pedestrian Head Kinematic and Injury Reconstruction}

The multi-body modeling method applied by the MADYMO software (MADYMO, 2013b) was used for kinematic reconstruction 

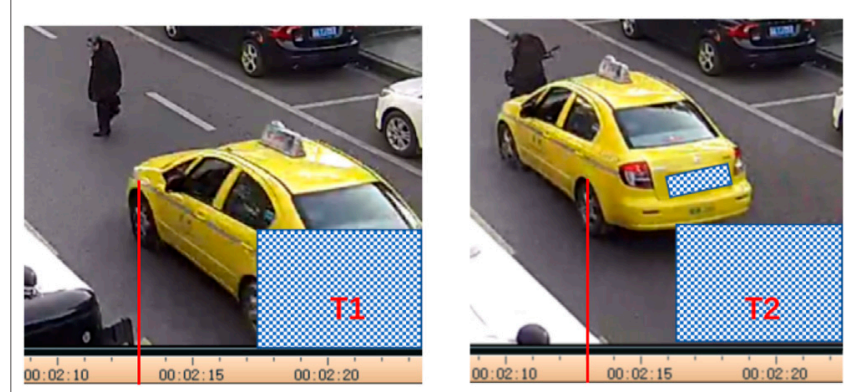

FIGURE 1 | Videos captured for estimation of vehicle impact speed and pedestrian posture.

of pedestrians involved in the 57 selected cases, where MADYMO pedestrian models (MADYMO, 2013a) scaled to the height and weight of the victims were employed. Similar to previous reconstruction work (Nie et al., 2014; Peng et al., 2013; Wu et al., 2021), each vehicle model was developed based on the geometric data measured from the blueprint or real car of the corresponding accident vehicle, and stiffness characteristics of the components were obtained from impactor test data reported in previous studies (Mizuno et al., 2001; Martinez et al., 2007) according to the available rating results of the vehicle model in subsystem tests.

For the cases with video data, the pedestrian posture and vehicle impact speed at the instant of vehicle-to-pedestrian contact were firstly estimated from the video. Figure 1 shows a sample, where the vehicle impact speed was calculated as the result of the wheelbase ( $2.5 \mathrm{~m}$ as measured) divided by the time between the video frame T1 and T2 (number of frames passed/ frame rate $=4 / 25 \mathrm{fps}=0.16 \mathrm{~s}$ ); thus, the vehicle impact speed was $15.63 \mathrm{~m} / \mathrm{s}(2.5 \mathrm{~m} / 0.16 \mathrm{~s}=15.63 \mathrm{~m} / \mathrm{s})$ for this case. For the cases without video data, the impact speed was estimated initially based on the data kinetic energy theorem calculation $\left(v=3.6^{\star} 2 \mu \mathrm{g} / \mathrm{L}^{2}: v\right.$ is the impact speed in $\mathrm{km} / \mathrm{h}, \mu$ is the friction coefficient, $g=9.8 \mathrm{~m} /$ $\mathrm{s}^{2}$, and $L$ is the breaking distance) and interview with the drivers.

For each case, a basic multi-body simulation of vehicle-topedestrian impact was then developed based on the estimated initial impact configuration (vehicle impact speed and pedestrian initial posture and contact position). Since the initial posture (gait stance, orientation, body bending state, etc.) of pedestrian at the instant of vehicle contact is one of the important factors affecting pedestrian kinematics and injury outcome (Untaroiu et al., 2009; Elliott et al., 2012; Chen et al., 2015). For the cases with video data, we defined the initial pedestrian posture in the simulations according to that in the video, while for cases without video data, pedestrian orientation and gait stance were defined according to the accident data (orientation was recorded by clock direction, and gait stance was simply recorded as struck leg forwarding or lagging) recorded by the accident investigation team after analysis of onsite, interview, injury location, and vehicle deformation information. Then, an optimization study using the framework shown in Figure $\mathbf{2}$ was conducted to find the optimal parameters of initial impact configuration for kinematic reconstruction.

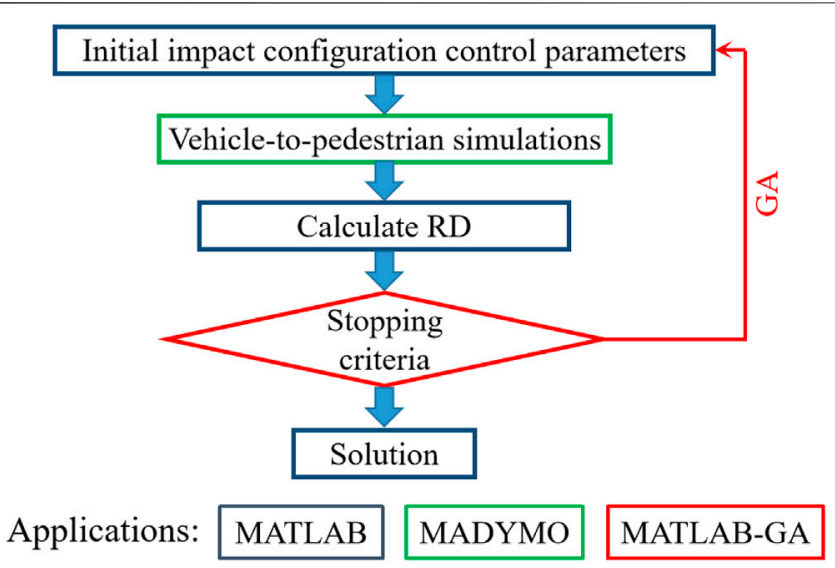

FIGURE 2 | Optimization framework for kinematic reconstruction.

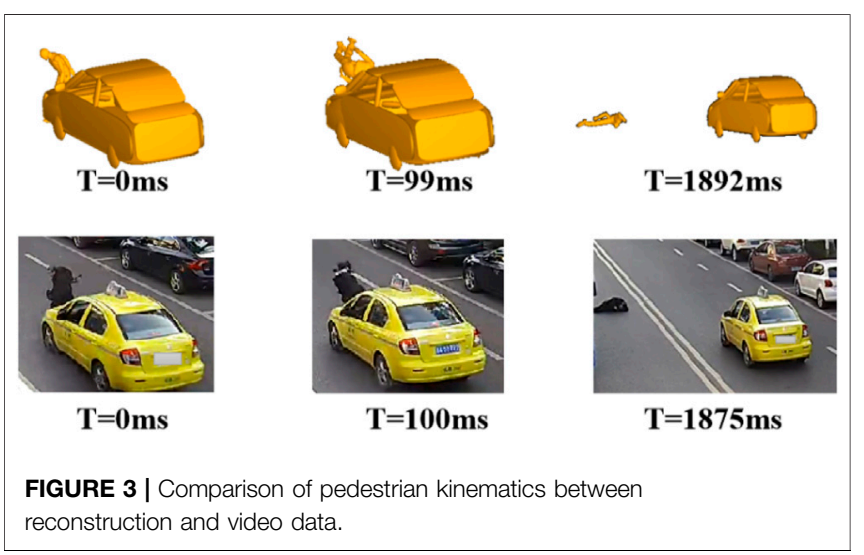

In the optimization, similar to the previous study ( $\mathrm{Li}$ et al., 2017b; Li et al., 2020), the Genetic Algorithm (GA) defined in the MATLAB Global Optimization Box was used to find the optimal solution, and the stopping criterion was defined as the maximum of 15 generations. Similar to the study of Untaroiu et al. (2009), the fitness function was defined so as to minimize the sum of relative distance $(\mathrm{RD})$ for each pedestrian contact location between prediction from the simulation and observation of the real-world case, which is calculated as follows:

$$
R D=\sum R D_{i}+R D_{\text {final }},
$$

where $R D_{i}$ is the relative distance for each observable pedestrian contact location on the vehicle and $R D_{\text {final }}$ is the relative distance of the final position of the pedestrian. For the cases without video data, variation ranges of $\pm 10 \mathrm{~km} / \mathrm{h}, \pm 30^{\circ}, \pm 100 \mathrm{~mm}$, and 10 different gait stances were set in the optimization study for vehicle impact speed, and pedestrian initial orientation, contact position, and gait stance, respectively.

Finally, a kinematic reconstruction simulation was carried out using the optimal parameters of initial impact configuration for each case. Figure 3 shows a sample of comparison between the predicted pedestrian kinematics from the reconstruction and the video data, where the 

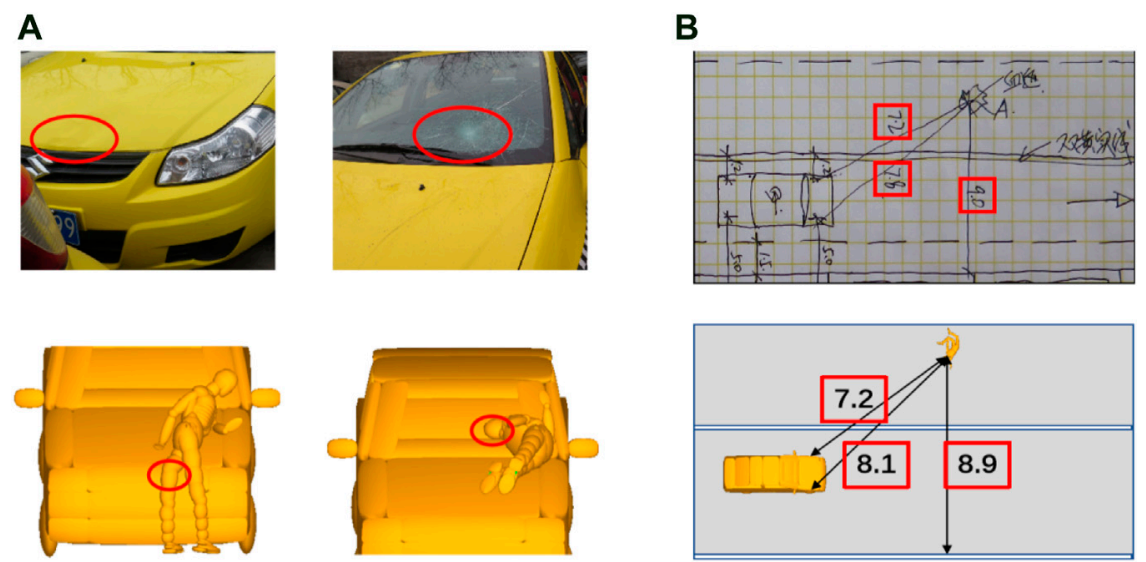

FIGURE 4 | Comparison of pedestrian contact location (A) and final position (B) between reconstructions and real-world cases.

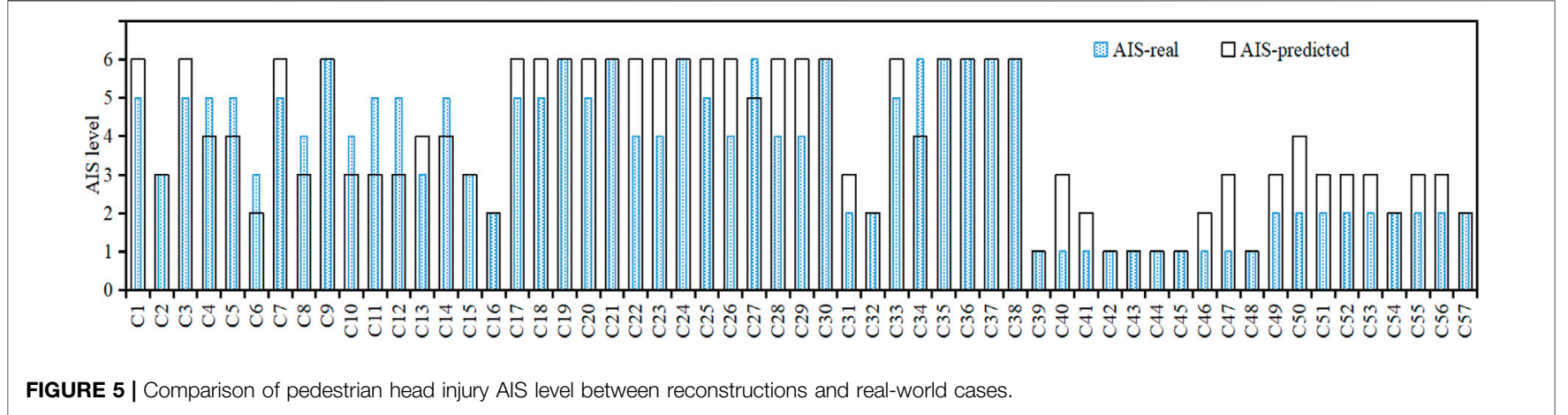

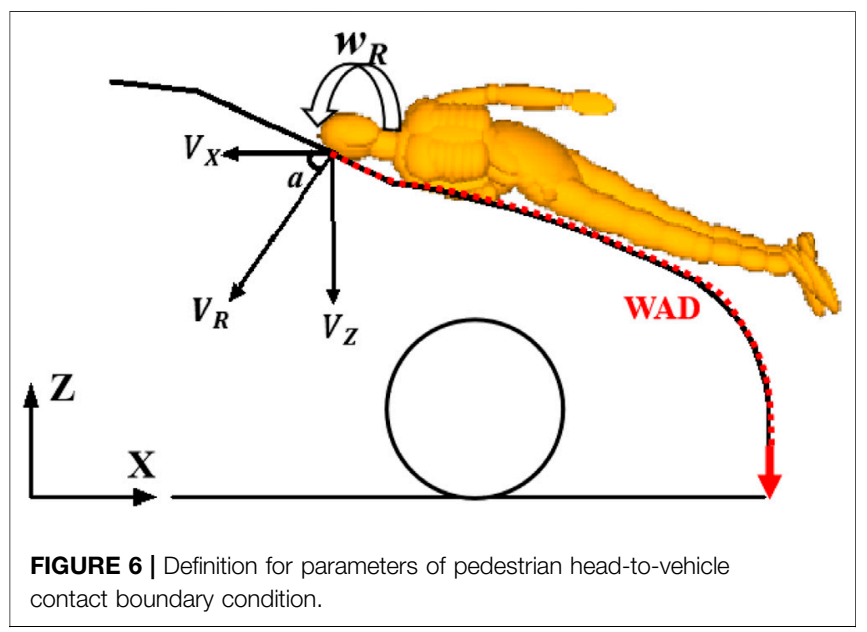

predicted specific pedestrian kinematics (leg-to-vehicle contact, head-to-vehicle contact, and pedestrian-to-ground contact) and corresponding time from the reconstruction simulation can generally match the video data. Figure 4 shows a sample of comparison between the predicted pedestrian contact locations and those on the accident vehicle; again, a good match can also be observed. To evaluate the stiffness assumptions, preliminary analysis was carried out to compare the AIS level of head injuries between the reconstructions and real-world cases (see Figure 5), where the predicted AIS levels were calculated from the predicted HIC using the regression models proposed by the NHTSA (National Highway Traffic Safety Administration) with a cut line of an injury risk of greater than 50\% (NHTSA, 1995). Generally, the predicted AIS level shows a good match with the real-world accident data. These indicate that pedestrian kinematic and injury reconstruction using multi-body modeling is basically plausible.

\section{Parameters of Pedestrian Head-to-Vehicle Contact Boundary Condition and Injury Criteria}

In the current work, pedestrian head-to-vehicle contact boundary conditions (Figure 6), including linear contact velocity $\left(V_{R}\right.$ : the resultant head linear velocity relative to the vehicle at the instant of head contact), angular contact velocity $\left(\boldsymbol{w}_{R}\right.$ : the resultant head rotational velocity around its center of gravity at the instant of head contact), contact angle ( $\boldsymbol{a}$ : the angle of linear contact velocity relative to the 

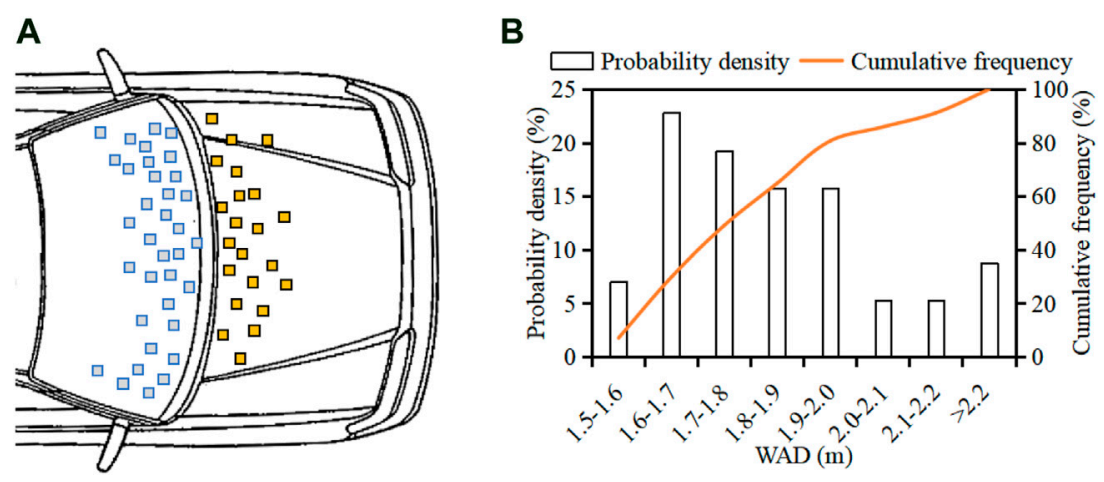

FIGURE 7 | Distribution of pedestrian head-to-vehicle contact location (A) and WAD (B)

horizontal plane), WAD (Wrap Around Distance), head contact time (time period between pedestrian initial contact and the instant of head contact), were extracted from simulations of pedestrian kinematic reconstruction. The predicted head kinematics of linear and angular acceleration and velocity extracted from the 57 reconstructions was employed to calculate the kinematicbased head injury criteria of HIC, HIP, GAMBIT, RIC, and BrIC according to the formulations introduced in Supplementary Appendix Information S1.

\section{Statistical Analysis}

Similar to previous studies (Shang et al., 2017; Li et al., 2018), the Mann-Whitney $U$-test, which is suitable for normally distributed and non-normally distributed data, was used to compare the difference in the distribution of a given parameter between two different groups (such as bonnet vs. windscreen impacts and sedan vs. SUV/MPV crashes), where the $p$-value was employed to indicate the statistical significance level of a comparison. The linear regression model was employed to analyze the relationship between two parameters with an $R^{2}$ value to assess the fitness level. The Weibull model was used to build the risk curves of serious head injury (AIS3+) as functions of the above kinematicbased head injury criteria similar to previous studies (Kerrigan et al., 2004; Takhounts et al., 2011 and 2013), where the probability of AIS3 + head injury as a function of a given criterion is given by

$$
P_{\mathrm{AIS} 3+}=1-e^{-(x / a)^{b}}
$$

where $x$ is the predictor, $a$ and $b$ are coefficients calculated from Weibull fitting. The AUC (Area Under the Curve) value with a threshold of 0.5 was employed to evaluate the predictive capability of the Weibull models similar to a previous study (Wu et al., 2021). The AUC can be calculated by two measures, namely, false-positive rate (FPR) and true-positive rate (TPR), where FPR refers to the ratio of false-positive cases (the cases that were predicted positive but are actually negative) out of all negative cases and TPR refers to the ratio of true-positive cases (the cases that were predicted positive and are actually positive too) out of all positive cases.

\section{RESULTS}

\section{Pedestrian Head-to-Vehicle Contact Boundary Condition}

Figure 7 shows the distribution of pedestrian head contact location and WAD. The data indicate that for $22(38.6 \%)$ and 35 (61.4\%) cases, the head contact location is on the bonnet and the windscreen, respectively. The reconstruction results show that head WAD values are in the range $1.5-2.3 \mathrm{~m}$ with an average of $1.84 \mathrm{~m}$ and that about $74 \%$ of cases have a WAD in the range $1.6-2.0 \mathrm{~mm}$.

Figure 8 shows the distribution and cumulative frequency of pedestrian head linear contact velocity, angular contact velocity, contact time, and contact angle. Pedestrian head linear contact velocity is mainly in the range $26-45 \mathrm{~km} / \mathrm{h}(67 \%)$, and $70 \%$ of cases have a head linear contact velocity below $40 \mathrm{~km} / \mathrm{h}$. The head angular contact velocity is mainly in the range $21-40 \mathrm{rad} / \mathrm{s}$ (67\%), and $80 \%$ of cases are with a head angular contact velocity lower than $40 \mathrm{rad} / \mathrm{s}$. More than half of the cases (58\%) have a head contact time in the period $80-140 \mathrm{~ms}$, and the head contact time is generally earlier than $140 \mathrm{~ms}(77 \%)$. The head contact angle is mainly in the range $40-70^{\circ}(70 \%)$, with an average value of $53^{\circ}$.

Figure 9 compares the distribution of pedestrian head linear contact velocity, angular contact velocity, contact time, and contact angle between bonnet and windscreen impacts. Generally, the head linear velocity and angular contact velocity in windscreen impacts are higher than those of bonnet impacts, but the differences are not statistically significant $(p>0.05)$. No obvious difference in head contact time was observed between bonnet and windscreen impacts, but the head contact angles for windscreen impacts are significantly bigger than those of bonnet impacts $(p=0.003)$. Figure 10 compares the distribution of pedestrian head linear contact velocity, angular contact velocity, contact time, and contact angle between sedan and SUV/MPV crashes. The SUV/MPV crashes have a significantly higher head angular contact velocity and earlier head contact time than collisions with sedans $(p<0.05)$, but no obvious differences in 

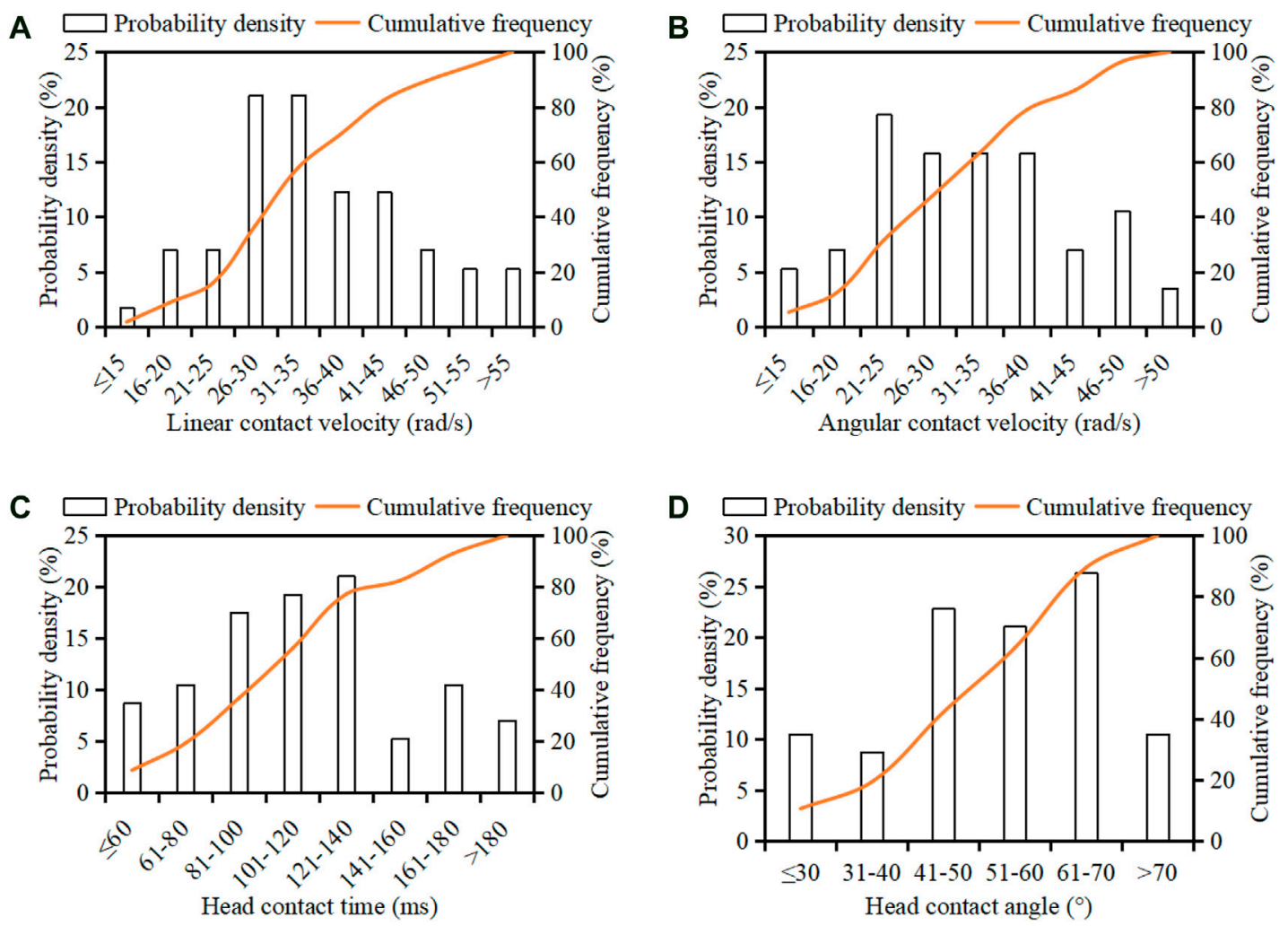

FIGURE 8 | Distribution of pedestrian head linear contact velocity (A), angular contact velocity (B), contact time (C), and contact angle (D).

head linear contact velocity and contact angle were observed between sedan and SUV/MPV crashes.

A further analysis was conducted to understand the relationship between pedestrian head-to-vehicle contact boundary condition and vehicle impact velocity. Figures 11A,B show the ratio of pedestrian head linear and angular contact velocity to vehicle impact velocity for each reconstructed case, together with the average value and standard deviation (SD) range for the sample. It should be noted that the ratio of head angular contact velocity to vehicle impact velocity is in the unit of $\mathrm{rad} / \mathrm{s}$ over $\mathrm{km} / \mathrm{h}$. Pedestrian head linear contact velocity is on average $83 \%$ (SD range: $60 \%-106 \%$ ) of the vehicle impact velocity, while the head angular contact velocity (in $\mathrm{rad} / \mathrm{s}$ ) is on average $75 \%$ (SD range: $50 \%-100 \%$ ) of the vehicle impact velocity in $\mathrm{km} / \mathrm{h}$. Figures 11C,D show the relationships between pedestrian head contact time and WAD/ height ratio and vehicle impact velocity for the bonnet-type (sedan and SUV) cars, where the cases involving MPVs were not included as their flatter front end can significantly affect pedestrian kinematics. Clearly, negative and positive linear correlations are observed for the relationships between pedestrian head contact time and $\mathrm{WAD} /$ height ratio and vehicle impact velocity, respectively.

\section{Pedestrian AIS3+ Head Injury Risk}

Figure 12 compares the AIS3+ head injury odds (the number of cases with AIS3+ injuries over the number of all cases) between bonnet and windscreen impacts as well as between sedan and SUV/MPV crashes. Clearly, the AIS3+ head injury odds are higher for impacts with bonnet (0.73) than for those with windscreen (0.63) and also higher for SUV/MPV crashes (0.83) than for sedan cases (0.51).

Supplementary Appendix Figure S1 shows the predicted HIC, HIP, GAMBIT, RIC, and BrIC for each case, where the magnitudes of the AIS1-2 group are obviously lower than that of the AIS3+ group for all criteria. Figure 13 shows pedestrian AIS3+ head injury risk curves as functions of HIC, HIP, GAMBIT, RIC, and BrIC estimated using the Weibull model and the data shown in Supplementary Appendix Figure S1. Table 1 presents the coefficients $a$ and $b$ in Eq. 2 and the AUC value for each Weibull model, where the AUC values are generally higher than $75 \%$ and can prove the good predictive capability of the Weibull models. Taking the 50\% AIS3+ head injury risk as the cut line, the thresholds are 1,300 for HIC, $60 \mathrm{~kW}$ for HIP, 0.74 for GAMBIT, $1,470 \times 10^{4}$ for RIC, 0.56 for BrIC2011, and 0.57 for BrIC2013. The predictions of the current work were compared with previous studies focusing on the thresholds for $50 \%$ risk of different head injuries in Table 2.

The relationships between HIC and other kinematic-based criteria analyzed using linear regression are shown in Figure 14, where the GAMBIT and RIC show a higher linear correlation to the HIC than to the HIP and BrIC, and the linear fit between BrIC2013 and HIC has the lowest $R^{2}$ value of all the correlations. 

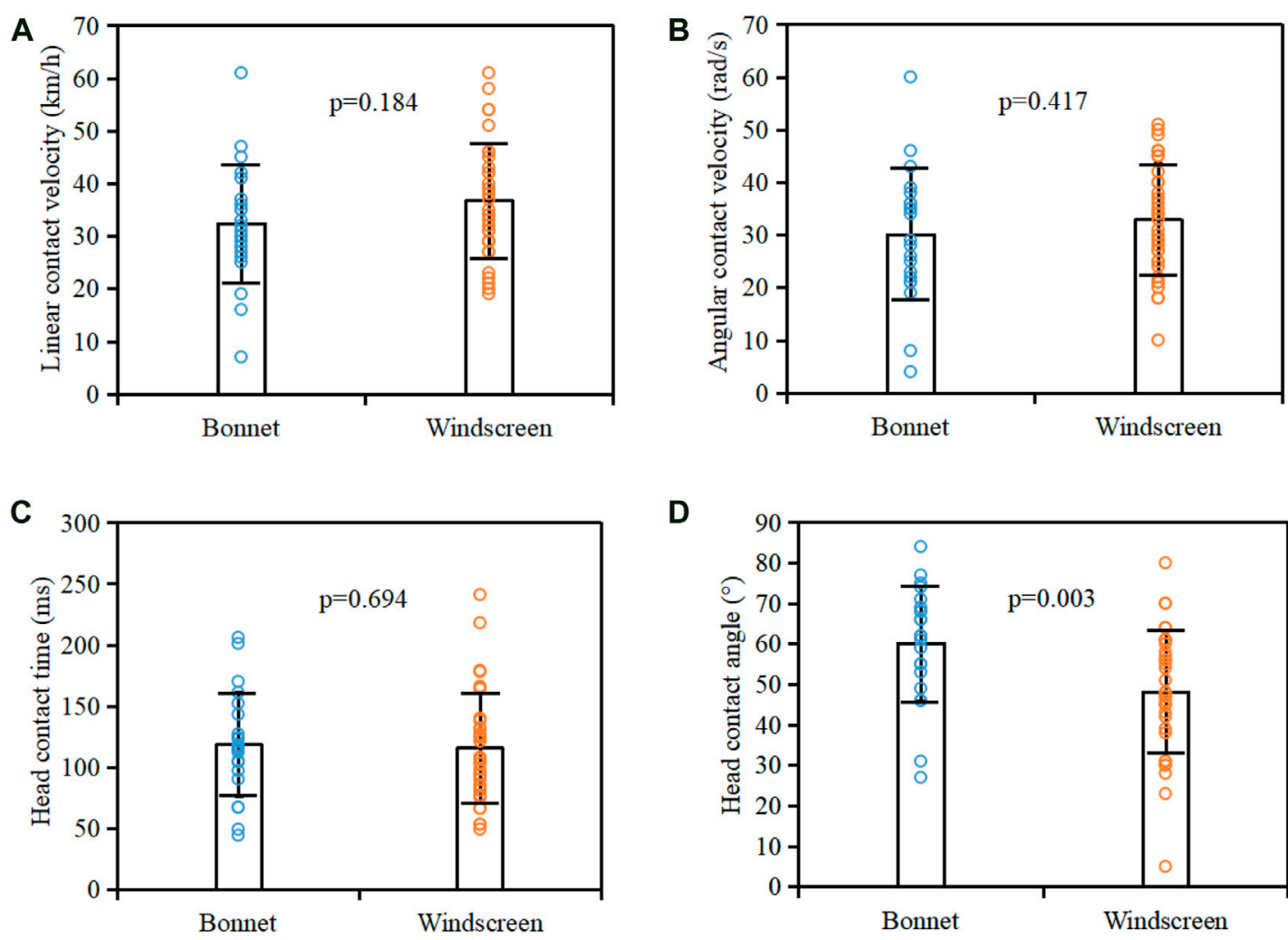

D

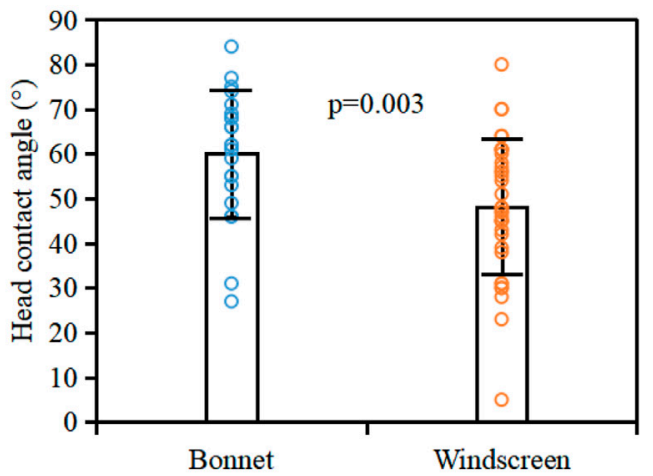

FIGURE 9 | Comparison of head linear contact velocity (A), angular contact velocity (B), contact time (C), and contact angle (D) between bonnet and windscreen impacts.

\section{DISCUSSION}

\section{Reference for Setup of Pedestrian Head-to-Vehicle Impact Boundary Condition}

The current work presents the data of Chinese adult pedestrian head-to-vehicle contact boundary condition from kinematic reconstruction of real-world collisions. The results show that head-to-windscreen contacts (61.4\%) are dominant in pedestrian collisions of the analysis sample, and head contact points cover the WAD range $1.5-2.3 \mathrm{~m}$ with $74 \%$ of cases in the range $1.6-2.0 \mathrm{~m}$ and an average value of $1.84 \mathrm{~m}$ (Figure 7B). The observed average WAD value from the current work is similar to that reported by Nie et al. (2014) based on the analysis of 21 cases from Changsha city of China $(1.82 \mathrm{~m})$, but distinguished with that from Germany data (Kiuchi et al., 2015) and the mixed data of China and Germany (Peng et al., 2013), where the average $\mathrm{WAD}$ is over $2.0 \mathrm{~m}$. The smaller WAD of Chinese pedestrians compared to that of Germans is mainly due to the shorter height of the Chinese population. These findings may suggest that the windscreen is an important part for pedestrian head protection for vehicle safety assessment in the Chinese market and that a closer focus should be paid on the area within a WAD of $1.6-2.0 \mathrm{~m}$, which is the main overlap range of the bonnet and windscreen (Figure 7), and legislative regulations should consider local characteristics. However, in the current C-IASI and C-NCAP, the adult head impactor test area for pedestrian protection mainly focuses on the WAD range 1.7-2.1 m (C-IASI, 2017; C-NCAP, 2020), so about $30 \%$ of adult head contacts on the vehicle observed in the current study are not included (Figure 7).

For head linear contact velocity, the current C-NCAP and C-IASI use $40 \mathrm{~km} / \mathrm{h}$ in the subsystem tests (C-IASI, 2017; C-NCAP, 2020). However, as observed in the current work (Figure 8A), this value can cover only $70 \%$ cases of the current sample. If coverage of $80 \%$ of cases is considered (regarded as the cut line for legislation similar to the EEVC, 2002), the head linear contact velocity should be $45 \mathrm{~km} / \mathrm{h}$ as observed here (Figure 8A). This implies that the current C-NCAP and C-IASI may overestimate the safety performance of vehicles in rear-world collisions given the recognized positive correlation between head linear impact velocity and pedestrian injury risk (Wang et al., 2020). For head angular contact velocity, the reconstruction results show that more than $67 \%$ of cases have a head angular contact velocity in the range $20-40 \mathrm{rad} / \mathrm{s}$ (Figure 8B). If coverage of $80 \%$ of cases is similarly considered, the head angular contact velocity is $40 \mathrm{rad} / \mathrm{s}$ (Figure 8B). The simulation data of the current work also indicate that the MPVs and SUVs could lead to a higher angular head contact velocity for pedestrians compared with sedans (Figure 10B); this trend is similar to that of cadaver 

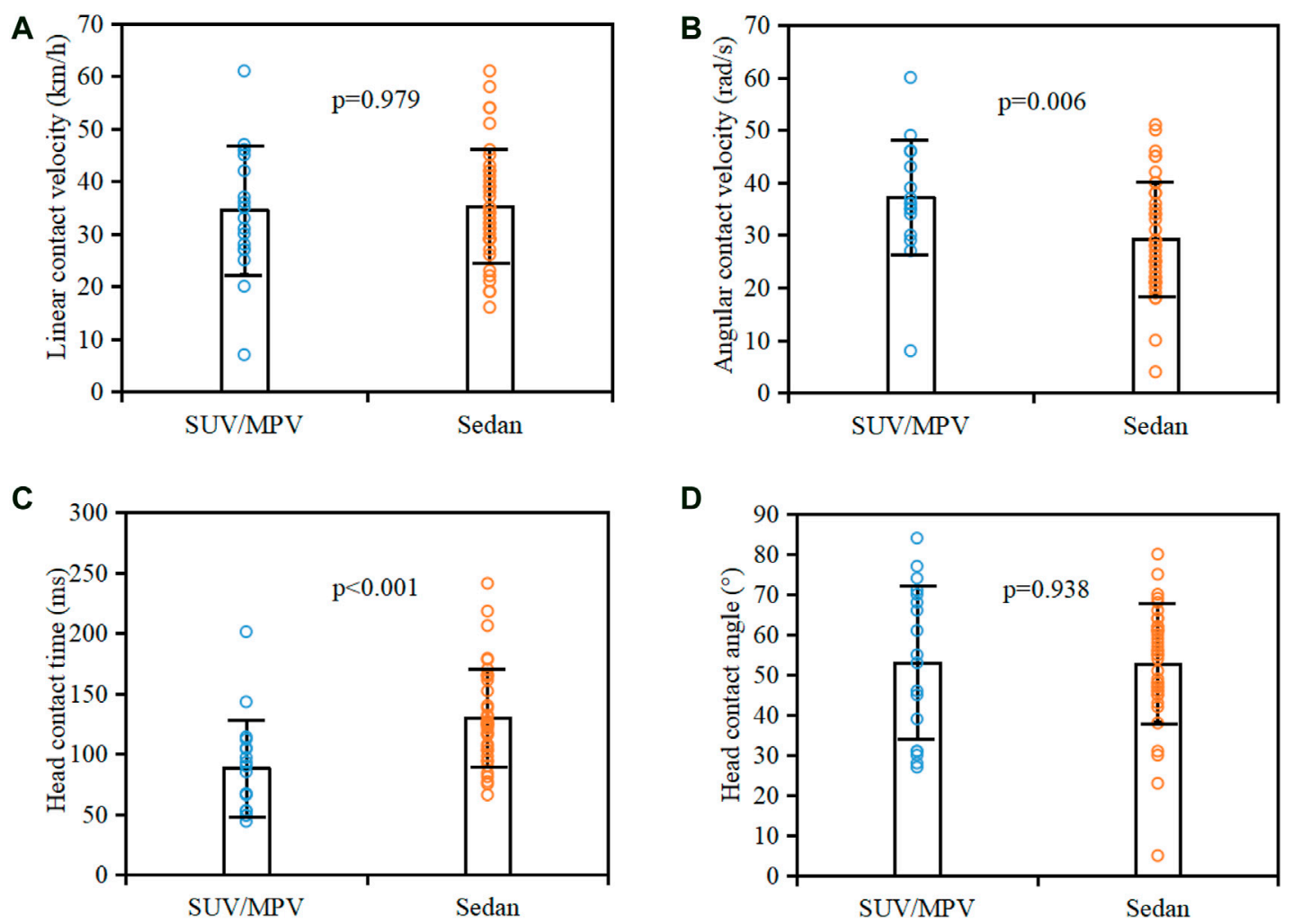

FIGURE 10 | Comparison of head linear contact velocity (A), angular contact velocity (B), contact time (C), and contact angle (D) between sedan and SUV/MPV crashes.

and dummy tests (Kerrigan et al., 2009) and may be largely due to the stronger upper body rotation caused by the higher bonnet leading edge of MPVs and SUVs. Many previous studies have indicated that head rotational motion has a significant influence on brain response and injury risk (Gennarelli et al., 1971; Rowson et al., 2011; Takhounts et al., 2011 and 2013). However, all current pedestrian safety regulations have not considered this impact boundary parameter in the subsystem tests. The above data could provide reference for future pedestrian safety regulations to consider the effect of head angular motion. It should be noted that the EEVC proposed the impact velocity for impactor tests according to vehicle impact velocity rather than the contact velocity of the corresponding body part, while the head impact boundary condition suggested by the current work was based on the observed head-to-vehicle contact data. On the other hand, the big gap between head-to-vehicle contact velocity and vehicle impact speed (Figures 11A,B) may imply that the impact velocity for head impactor tests directly sourced from the head-to-vehicle contact boundary condition might be more reasonable. Furthermore, the ratio of respectively $83 \%$ (SD range: $60-106 \%$ ) and $75 \%$ (SD range: $50-100 \%)$ for pedestrian head linear $(\mathrm{km} / \mathrm{h})$ and angular $(\mathrm{rad} / \mathrm{s})$ contact velocity to vehicle impact velocity $(\mathrm{km} / \mathrm{h})$ observed in the current work (Figures 11A,B) could provide reference for estimation of pedestrian head-to-vehicle contact velocity from vehicle initial impact speed.
The results indicate that $70 \%$ of cases have a head contact angle in the range $40-70^{\circ}$, and the average value is $53^{\circ}$ (Figure 8D). This finding is similar to that of $\mathrm{Nie}$ et al. (2014), but the average value is $12^{\circ}$ less than the $65^{\circ}$ defined in C-NCAP and C-IASI. Given the dramatic difference in the inclination angle between windscreen $\left(23-55^{\circ}\right.$, median $\left.=30^{\circ}\right)$ and bonnet $\left(8-37^{\circ}\right.$, median $\left.=13^{\circ}\right)$ (Li et al., 2017a), the same head linear contact velocity at a certain incident angle to the bonnet and windscreen will lead to an obviously different perpendicular impact velocity to the head (Figure 15), which is the principal component for the head impact load. Moreover, Figure 9D indicates that the head contact angle for the cases with bonnet impacts (average $=60^{\circ}$ ) is significantly higher than that for windscreen impacts (average $\left.=48^{\circ}\right)$. These findings may suggest that the current C-NCAP and C-IASI might need to reduce the adult head contact angle in the test procedures and to distinguish the bonnet and windscreen.

Pedestrian head contact time and WAD (determining the contact location) are important factors for pressure and structure control in the development of pedestrian airbag (Lim et al., 2015). The kinematic reconstruction data show that the head contact time is mainly (77\%) in the range of 50-140 ms (Figure 8C), which is affected by vehicle type with a significantly earlier head contact time in SUV/MPV crashes than in collisions with sedans (Figure 10C) and also affected by vehicle impact velocity by a negative linear correlation 

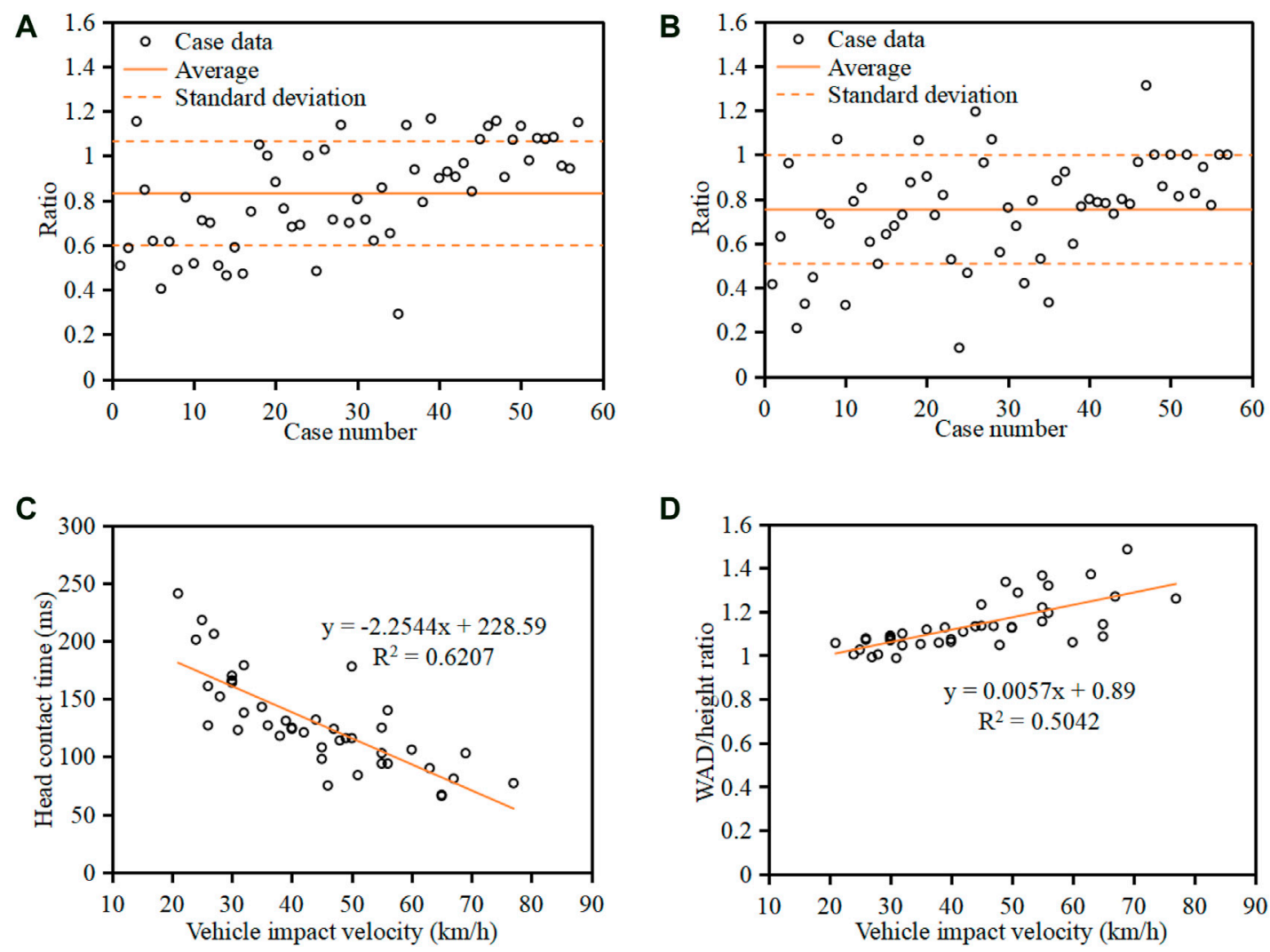

FIGURE 11 | Ratio of head linear contact velocity in $\mathrm{km} / \mathrm{h}$ (A) and angular contact velocity in $\mathrm{rad} / \mathrm{s}$ (B) to vehicle impact velocity in $\mathrm{km} / \mathrm{h}$ and relationships between pedestrian head contact time (C) and WAD/height ratio (D) and vehicle impact velocity for the bonnet-type (sedan and SUV) cars.
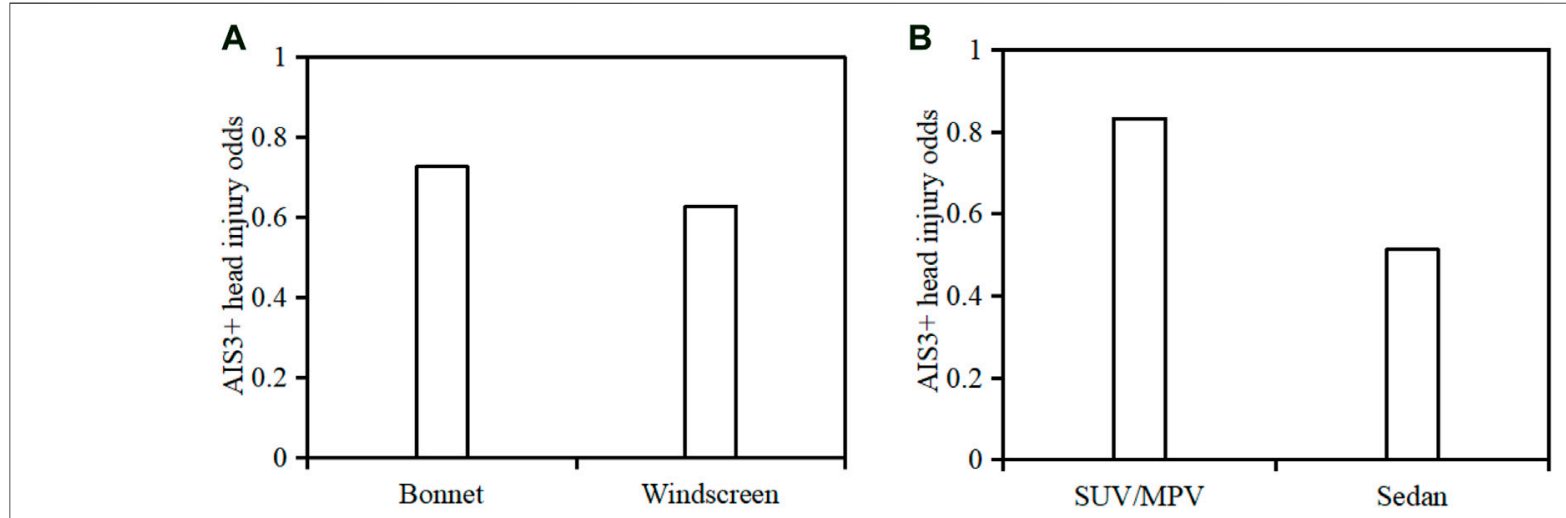

FIGURE 12 | Comparison of AIS3+ head injury odds between bonnet and windscreen impacts (A) and between sedan and SUV/MPV crashes (B)

(Figure 11C). The results also show that the WAD is influenced by pedestrian height and vehicle impact velocity, where a positive linear correlation is observed for the relationship between pedestrian WAD/height ratio and vehicle impact velocity (Figure 11D). These findings could provide evidence for estimation of pedestrian head contact time and location from accident scenarios.

\section{Reference for Estimation of Pedestrian Head Injury Risk}

The predicted thresholds of HIC, HIP, GAMBIT, RIC, BrIC2011, and BrIC2013 for a $50 \%$ probability of AIS3 + head injury risk are $1,300,60 \mathrm{~kW}, 0.74,1,470 \times 10^{4}, 0.56$, and 0.57 , respectively (Figure 13). The comparisons in Table 2 indicate that the HIC of 1,300 for $50 \%$ AIS3+ head injury risk observed in the 


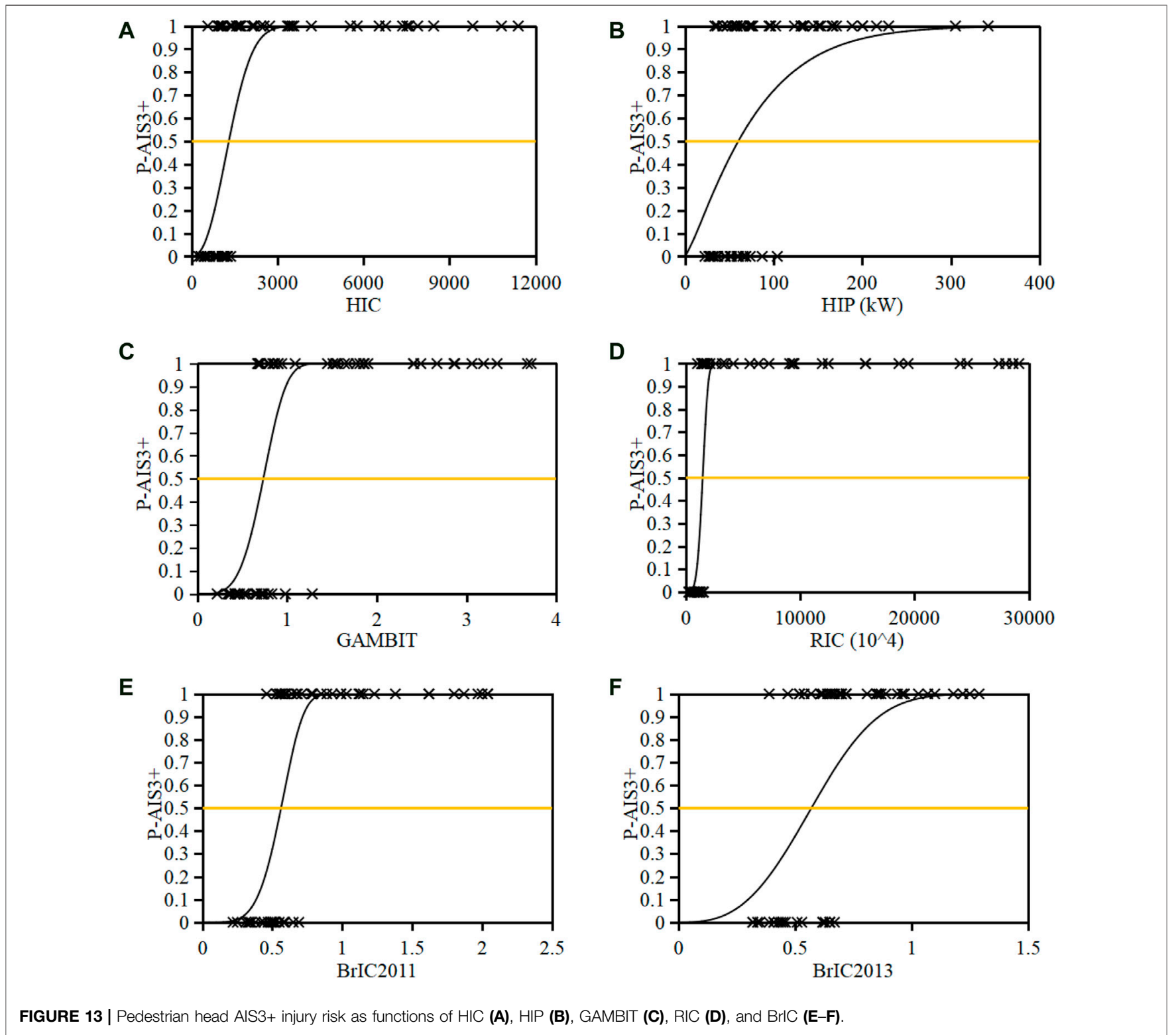

TABLE 1 | Weibull model results for HIC, HIP, GAMBIT, RIC, and BrIC.

\begin{tabular}{lccccc} 
Predictor & HIC & HIP & GAMBIT & RIC & BrIC2011 \\
\hline a & $1,519.013$ & 81.359 & 0.803 & $1,593.119$ & 0.606 \\
b & 2.321 & 1.188 & 4.110 & 4.343 & 0.963 \\
AUC & $84.2 \%$ & $64.8 \%$ & $84.2 \%$ & $84.2 \%$ & $84.2 \%$
\end{tabular}

current work is similar to that of 1,442 from a previous reconstruction study of pedestrian collisions (Peng et al., 2013), that of 1,450 for $50 \%$ of skull fracture reported by Mertz et al. (2003) in the analysis of the data from Injury Assessment Reference Values, and that of 1,429 for $50 \%$ subdural hematoma risk predicted from reconstructions of mixed data of motorcyclist, footballer, and pedestrian accidents using an isolated human body head FE model (Marjoux et al., 2008). However, the HIC of 1,300 is much higher than that of 667 for a 50\% skull fracture risk and 1,032 for a $50 \%$ severe neurological injury risk observed in the study of Marjoux et al. (2008). The HIP of $60 \mathrm{~kW}$ for $50 \%$ AIS3+ head injury risk predicted here is higher than that of $38 \mathrm{~kW}$ for $50 \%$ skull fracture risk and $48 \mathrm{~kW}$ for $50 \%$ severe neurological injury 
TABLE 2 | Thresholds for 50\% risk of head injuries from different studies.

\begin{tabular}{|c|c|c|c|c|c|c|c|}
\hline Study & Head injury & HIC & HIP (kW) & GAMBIT & RIC & BrIC2011 & BrIC2013 \\
\hline Current work & AlS3+ & 1,300 & 60 & 0.74 & $1,470 \times 10^{4}$ & 0.56 & 0.57 \\
\hline Peng et al. (2013) & AlS3+ & 1,442 & - & - & - & - & - \\
\hline Mertz et al. (1996) & Skull fracture & $1,400-1,600$ & - & - & - & - & - \\
\hline Mertz et al. (2003) & Skull fracture & 1,450 & - & - & - & - & - \\
\hline \multirow[t]{3}{*}{ Marjoux et al. (2008) } & Skull fracture & 667 & 38 & - & - & - & - \\
\hline & Severe neurological injury & 1,032 & 48 & - & - & - & - \\
\hline & Subdural hematoma & 1,429 & 55 & - & - & - & - \\
\hline Newman (1986) & AlS3+ & - & - & 1.0 & - & - & - \\
\hline Kimpara and Iwamoto (2012) & Mild TBI & - & - & - & $1,030 \times 10^{4}$ & - & - \\
\hline Takhounts et al. (2011) & AIS3+ TBI & - & - & - & - & 1.0 & - \\
\hline Takhounts et al. (2013) & AlS3+ TBI & - & - & - & - & - & $0.95 / 0.87$ \\
\hline
\end{tabular}

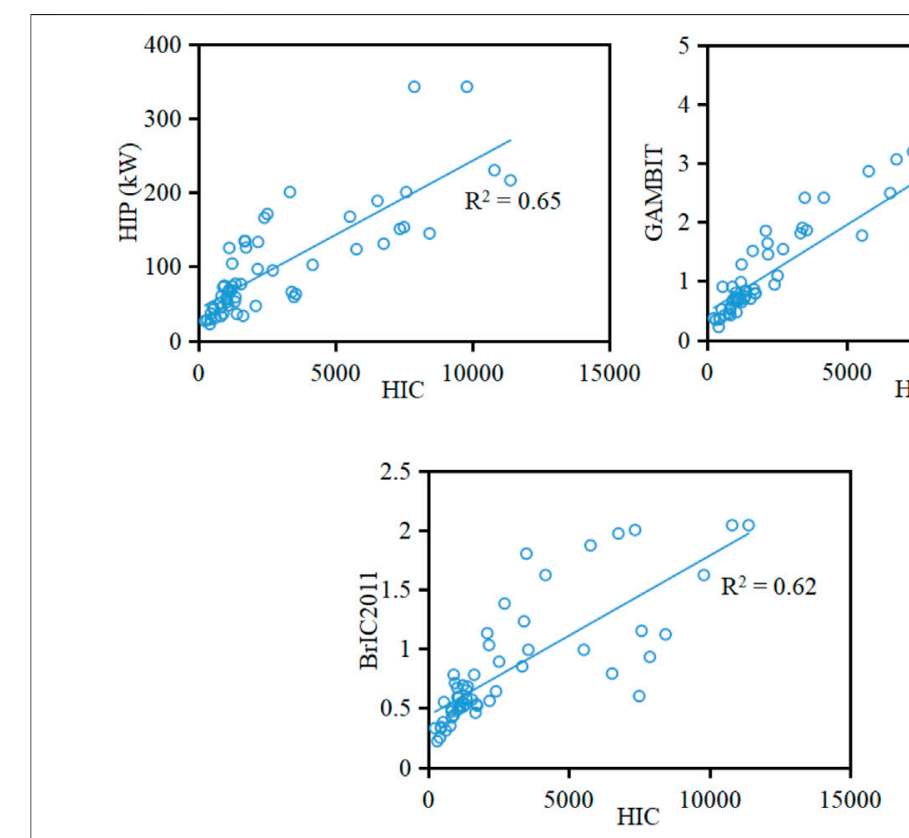

FIGURE 14 | Linear regression between HIC and HIP, GAMBIT, RIC, and BrIC.

risk, but similar to that of $55 \mathrm{~kW}$ for $50 \%$ subdural hematoma risk observed from Marjoux et al. (2008). The GAMBIT of 0.74 for $50 \%$ AIS3 + head injury risk predicted by the current work is a little lower than that of 1.0 proposed by Newman (1986). The RIC of $1,470 \times 10^{4}$ for a $50 \%$ AIS3 + head injury risk estimated from current reconstruction data is obviously higher than that of 1,030 $\times 10^{4}$ for $50 \%$ mild TBI (AIS2) (Kimpara and Iwamoto, 2012), but no threshold is available in previous studies for AIS3+ head injuries. The predicted thresholds of BrIC2011 and BrIC2011 for a 50\% AIS3 + head injury risk are much lower than those reported in previous studies from reconstructions of dummy impact tests of vehicle occupants (Takhounts et al., 2011 and 2013).

The above analysis suggests that the thresholds for the same injury level and criterion may vary from study methods (isolated FE human body head modeling, multi-body full body modeling, tests of ATDs, etc.) and data sample (pedestrian, motorcyclist, footballer, and occupant accidents or mixed data). Generally, the thresholds of HIC, HIP GAMBIT, and RIC for a 50\% AIS3+ head injury risk predicted from current reconstruction data are plausible when compared to previous studies. However, the thresholds for BrIC2011 and BrIC2011 show a big gap with the data from Takhounts et al. (2011) and Takhounts et al. (2013). This difference might be due to the fact that the values of BrIC should be used in conjunction with the injury assessment device it is measured with as claimed by Takhounts et al. (2011) and Takhounts et al. (2013). In Euro-NCAP, C-NCAP, and C-IASI, the HIC of 650 and 1,700 are regarded as the thresholds for "ADEQUATE" and "POOR" performance of vehicle safety, respectively (C-IASI, 2017; C-NCAP, 2020; Euro-NCAP 2020). These cut lines were defined based on a specific risk of skull fracture observed from studies of biomechanics. Similarly, the predicted head injury risk curves of the current work could provide reference for this, considering various kinematic-based criteria HIC, HIP, GAMBIT, RIC, and BrIC. 


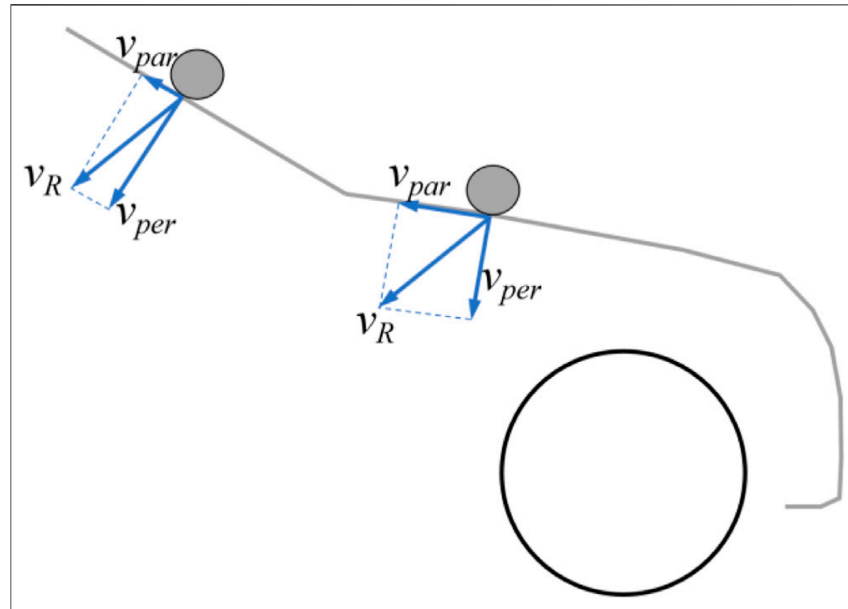

FIGURE 15 | Decomposition of head contact velocity in bonnet and windscreen impacts.

HIC is currently used in NCAPs for estimation of pedestrian injury risk, but other kinematic-based criteria could be potential supplements for the evaluation of pedestrian head injuries given the limitation of HIC. The linear correlation analysis between HIC and other kinematic-based criteria (Figure 14) indicates that HIC provides a higher correlation $\left(R^{2}>0.7\right)$ to GAMBIT and RIC than to HIP and BrIC. This is mainly due to the fact that the physical quantities considered in the formulations of HIC, GAMBIT, and RIC are head acceleration (linear and/or angular) (Supplementary Appendix Information S1), while HIP and BrIC include head angular velocity; particularly, BrIC2013 considers head angular velocity only and has the lowest $R^{2}$ value $(0.48)$ to HIC. This finding may suggest that HIC could generally represent the kinematic-based criteria considering acceleration only, such as the GAMBIT and RIC, but could not represent the criteria which include head angular velocity which could be considered as potential supplements to HIC for evaluation of pedestrian head injuries. On the other hand, the AUC values indicate that the predictive capability of the injury curves developed based on HIP (AUC $=64.8 \%$ ) and BrIC2013 (AUC $=73.7 \%$ ) is lower than that based on other kinematic-based criteria (AUC $=84.2 \%$ ) for the current analysis sample. The above findings may suggest that GAMBIT, RIC, and BrIC2011 could be considered as the potential supplements of HIC for evaluation of pedestrian head injuries.

\section{Limitations}

There are several limitations in the current study. Firstly, there are some uncertainties in the information of accident data, especially the estimated vehicle impact speed and initial posture of the pedestrian, which could have a significant influence on the reconstruction results, but the optimization process can help to reduce the estimation error. Secondly, the human body model for an individual case was scaled from the 50th\% adult male model, where characteristics of joints and stature could only be approximated and the age effect on the mechanical properties of human body was not considered in the model. Thirdly, stiffness curves of vehicle models were defined according to the available rating results of the vehicle models in subsystem tests and the corresponding stiffness curves published in previous studies since it is not able to access the test data for the exact vehicle model given the public unavailability of stiffness curves for vehicle models. This could lead to some uncertainties to the predicted magnitudes of head injury criteria. Although there are many shortcomings for this approach, this is one of the reasonable solutions for stiffness assumption and widely used in pedestrian crash reconstructions (Nie et al., 2014; Peng et al., 2012; Wu et al., 2021), and the good match in the AIS level between the predictions and real-world accident data (Figure 5) could support the reliability of this approach. Finally, the parameters such as contact friction coefficients were defined according to the literature, which also led to some uncertainties in the modeling. However, there are inherent drawbacks in the reconstruction study of real-world collisions (Huang er al., 2018; Nie et al., 2014; Penr et al., 2013), and further improvements in the availability of data information for accident scenarios, mechanical properties of the human body and vehicles, and the modeling approach are needed to overcome these difficulties.

\section{CONCLUSION}

This study is the first attempt at understanding the characteristics of Chinese pedestrian head-to-vehicle contact boundary condition considering rotational contact velocity and head injury risk as functions of the kinematic-based criteria HIC, HIP, GAMBIT, RIC, and BrIC from reconstructions of realworld collisions using full-body human body models. The findings of the current work could provide realistic reference for evaluation of vehicle safety performance focusing on pedestrian protection, which are summarized as follows:

(1) Head-to-windscreen contacts are dominant in pedestrian collisions of the analysis sample, and head WAD floats from 1.5 to $2.3 \mathrm{~m}$, with a mean value of $1.84 \mathrm{~m}$.

(2) Eighty percent of cases have a head linear contact velocity below $45 \mathrm{~km} / \mathrm{h}$ or an angular contact velocity less than $40 \mathrm{rad} / \mathrm{s}$; pedestrian head linear contact velocity is on average $83 \pm 23 \%$ of the vehicle impact velocity, while the head angular contact velocity (in $\mathrm{rad} / \mathrm{s}$ ) is on average $75 \pm$ $25 \%$ of the vehicle impact velocity in $\mathrm{km} / \mathrm{h}$.

(3) Seventy-seven percent of cases have a head contact time in the range $50-140 \mathrm{~ms}$, and negative and positive linear correlations are observed for the relationship between pedestrian head contact time and $\mathrm{WAD} /$ height ratio and vehicle impact velocity, respectively.

(4) Seventy percent of cases have head contact angle floats from 40 to $70^{\circ}$, with an average value of $53^{\circ}$; the pedestrian head contact angles on windscreens (average $=48^{\circ}$ ) are significantly lower than those on bonnets (average $=60^{\circ}$ ).

(5) AIS3 + head injury risk curves as functions of kinematicbased criteria HIC, HIP, GAMBIT, RIC, BrIC2011, and BrIC2013 were built, where the thresholds for a $50 \%$ 
probability of AIS3+ head injury risk are 1,300, $60 \mathrm{~kW}, 0.74$, $1,470 \times 10^{4}, 0.56$, and 0.57 , respectively.

(6) HIC provides a high linear correlation to the kinematic-based criteria considering acceleration only (GAMBIT and RIC), but a lower linear correlation to the criteria which include head angular velocity (HIP and BrIC).

\section{DATA AVAILABILITY STATEMENT}

The original contributions presented in the study are included in the article/Supplementary Material, further inquiries can be directed to the corresponding authors.

\section{AUTHOR CONTRIBUTIONS}

GL: analyze data and draft the work, JL: analyze and interpret data, KL: provide conception and draft the work, HZ: provide conception and approval of accident cases, LS: ensure questions

\section{REFERENCES}

Chen, H., Poulard, D., Crandall, J. R., and Panzer, M. B. (2015). "Pedestrian Response with Different Initial Positions during Impact with a Mid-sized sedan," in Proceedings of the 24th International Technical Conference of Enhanced Safety of Vehicles (Gothenburg, Sweden. ESV Paper No. 15-0391.

EEVC (2002). Improved Test Methods to Evaluate Pedestrian Protection Affordable by Passenger Cars, EEVC Working Group17 Report. Rome, Italy: European Enhanced Vehicle-Safety Committees.

Elliott, J. R., Simms, C. K., and Wood, D. P. (2012). Pedestrian Head Translation, Rotation and Impact Velocity: the Influence of Vehicle Speed, Pedestrian Speed and Pedestrian Gait. Accid. Anal. Prev. 45, 342-353. doi:10.1016/ j.aap.2011.07.022

Euro-Ncap (2020). Assessment Protocol-Vulnerable Road User Protection. Leuven, Belgium: European New Car Assessment Programme.

Gennarelli, T. A., Ommaya, A. K., and Thibault, L. E., 1971. Comparison of Translational and Rotational Head Motions in Experimental Cerebral Concussion.

Gennarelli, T. A., and Wodzin, E. (2006). AIS 2005: a Contemporary Injury Scale. Injury 37 (12), 1083-1091. doi:10.1016/j.injury.2006.07.009

Huang, J., Peng, Y., Yang, J., Otte, D., and Wang, B. (2018). A Study on Correlation of Pedestrian Head Injuries with Physical Parameters Using In-Depth Traffic Accident Data and Mathematical Models. Accid. Anal. Prev. 119, 91-103. doi:10.1016/j.aap.2018.07.012

C-Iasi (2017). Part 3: Pedestrian Safety Index, Pedestrian Protection Test Protocol, China Insurance Automotive Safety Index. Chongqing, China: China Automotive Engineering Research Institute.

Kerrigan, J., Arregui, C., and Crandall, J. (2009). "Pedestrian Head Impact Dynamics: Comparison of Dummy and PMHS in Small sedan and Large SUV Impacts," in Proceedings of the 21th International Technical Conference of Enhanced Safety of Vehicles (Stuttgart, Germany. ESV Paper No. 09-0127.

Kerrigan, J. R., Drinkwater, D. C., Kam, C. Y., Murphy, D. B., Ivarsson, B. J., Crandall, J. R., et al. (2004). Tolerance of the Human Leg and Thigh in Dynamic Latero-Medial Bending. Int. J. Crashworthiness 9 (6), 607-623. doi:10.1533/ijcr.2004.0315

Kimpara, H., and Iwamoto, M. (2012). Mild Traumatic Brain Injury Predictors Based on Angular Accelerations during Impacts. Ann. Biomed. Eng. 40 (1), 114-126. doi:10.1007/s10439-011-0414-2

Kiuchi, T., Lubbe, N., Otte, D., and Chen, Q. (2015). "Comparative Study of VRU Head Impact Locations," in Expert Symposium on Accident Research (Hannover, Germany. related to the accuracy of reconstruction, SZ: ensure questions related to the accuracy of reconstruction, JN: revise draft critically for important intellectual content.

\section{FUNDING}

This work was supported by the National Natural Science Foundation of China (Grant Nos. 51805162, 31800788, and 32171305), Scientific Research Foundation of Hunan Provincial Education Department (Grant Nos. 20B233 and 19C1559) and Natural Science Foundation of Hunan Province, China (Grant No. 2019JJ70045).

\section{SUPPLEMENTARY MATERIAL}

The Supplementary Material for this article can be found online at: https:/www.frontiersin.org/articles/10.3389/fbioe.2021.768994/ full\#supplementary-material

Li, G., Lyons, M., Wang, B., Yang, J., Otte, D., and Simms, C. (2017a). The Influence of Passenger Car Front Shape on Pedestrian Injury Risk Observed from German In-Depth Accident Data. Accid. Anal. Prev. 101, 11-21. doi:10.1016/ j.aap.2017.01.012

Li, G., Wang, F., Otte, D., Cai, Z., and Simms, C. (2018). Have Pedestrian Subsystem Tests Improved Passenger Car Front Shape? Accid. Anal. Prev. 115, 143-150. doi:10.1016/j.aap.2018.03.014

Li, G., Wang, F., Otte, D., and Simms, C. (2019). Characteristics of Pedestrian Head Injuries Observed from Real World Collision Data. Accid. Anal. Prev. 129, 362-366. doi:10.1016/j.aap.2019.05.007

Li, G., Yang, J., and Simms, C. (2017b). Safer Passenger Car Front Shapes for Pedestrians: a Computational Approach to Reduce Overall Pedestrian Injury Risk in Realistic Impact Scenarios. Accid. Anal. Prev. 100, 97-110. doi:10.1016/ j.aap.2017.01.006

Lim, J.-H., Park, J.-S., Yun, Y.-W., Jeong, S.-B., and Park, G.-J. (2015). Design of an Airbag System of a Mid-sized Automobile for Pedestrian protection. Proc. Inst. Mech. Eng. D: J. Automobile Eng. 229 (5), 656-669. doi:10.1177/ 0954407014551186

MADYMO (2013a). Human Body Models Manual Release, 7.5. Delft, Netherlands: TASS.

MADYMO (2013b). Madymo Theory Manual Release 7.5. Delft, Netherlands: TASS.

Marjoux, D., Baumgartner, D., Deck, C., and Willinger, R. (2008). Head Injury Prediction Capability of the HIC, HIP, SIMon and ULP Criteria. Accid. Anal. Prev. 40 (3), 1135-1148. doi:10.1016/j.aap.2007.12.006

Martinez, L., Guerra, J., Ferichola, G., Garcia, A., and Yang, J. (2007). "Stiffness Corridors of the European Fleet for Pedestrian Simulation," in Proceedings of the 20th Experimental Safety Vehicles Conference (Lyon, France. ESV Paper No. 07-0267.

Mertz, H. J., Irwin, A. L., and Prasad, P. (2003). Biomechanical and Scaling Bases for Frontal and Side Impact Injury Assessment Reference Values. Stapp Car Crash J. 47, 155-188. doi:10.4271/2003-22-0009

Mertz, H., Prasad, P., and Nusholtz, G. (1996). Head Injury Risk Assessment for Forehead Impacts, SAE Technical Paper No. 960099.

Mizuno, K., Yonezawa, H., and Kajzer, J. (2001). "Pedestrian Headform Impact Tests for Various Vehicle Locations," in Proceedings of the 17th International Technical Conference on the Enhanced Safety of Vehicles (ESV) (Amsterdam, Netherlands. ESV Paper No. 278.

Mizuno, Y. (2005). "Summary of IHRA Pedestrian Safety Working Group Activities-Proposed Test Methods to Evaluate Pedestrian protection Offered by Passenger Cars," in Proceedings of the 19th International Technical 
Conference on the Enhanced Safety of Vehicles (ESV) (Washington, USA. ESV Paper No. 05-0138-O.

C-Ncap (2020).C-NCAP Management Regulation (2021 Version), China New Car Assessment Programme Center. Tianjin, China: China Automotive Technology and Research Center.

J-Ncap (2014). Pedestrian Protection Performance Tests. Tokyo, Japan: Japan New Car Assessment Programme.

Newman, J. (1986). “A Generalized Acceleration Model for Brain Injury Threshold (GAMBIT)," in Proceedings of the International Research Council on Biomechanics of Injury (IRCOBI) Conference (Zurich, Switzerland.

Newman, J. A., Shewchenko, N., and Welbourne, E. (20002000). A Proposed New Biomechanical Head Injury Assessment Function - the Maximum Power index. Stapp Car Crash J. 44, 215-247. doi:10.4271/2000-01-sc16

NHTSA (1995). FMVSS No. 201, Upper interior Head protection. Washington, D.C: National Highway Traffic Safety Administration.

Nie, J., Li, G., and Yang, J. (2014). A Study of Fatality Risk and Head Dynamic Response of Cyclist and Pedestrian Based on Passenger Car Accident Data Analysis and Simulations. Traffic Inj. Prev. 16 (1), 76-83. doi:10.1080/15389588.2014.881477

Peng, Y., Deck, C., Yang, J., Otte, D., Willinger, R., Rowson, S., et al. (2013). A Study of Adult Pedestrian Head Impact Conditions and Injury Risks in Passenger Car Collisions Based on Real-World Accident dataRotational Head Kinematics in Football Impacts: An Injury Risk Function for Concussion. Traffic Inj. Prev. 1440 (61), 6391-4613. doi:10.1080/15389588.2012.733841

Schmitt, K. U., Niederer, P. F., Muser, M. H., and Walz, F. (2010). Trauma Biomechanics. Third edition. Berlin,Germany, Springer.

Shang, S., Otte, D., Li, G., and Simms, C. (2017). Detailed Assessment of Pedestrian Ground Contact Injuries Observed from In-Depth Accident Data. Accid. Anal. Prev. 110, 9-17. doi:10.1016/j.aap.2017.10.011

Simms, C., and Wood, D. (2009). Pedestrian and Cyclist Impact. Berlin,Germany, Springer.

Takhounts, E. G., Craig, M. J., Moorhouse, K., Mcfadden, J., and Hasija, V. (2013). Development of Brain Injury Criteria (BrIC). Stapp Car Crash J. 57, 243-266. doi:10.4271/2013-22-0010

Takhounts, E., Hasija, V., Ridella, S., Rowson, S., and Duma, S. (2011). "Kinematic Rotational Brain Injury Criterion (BrIC)," in Proceedings of the 22th Experimental Safety Vehicles Conference (Washington, DC: U.S.). ESV Paper No. 11-0263.

Untaroiu, C. D., Meissner, M. U., Crandall, J. R., Takahashi, Y., Okamoto, M., and Ito, O. (2009). Crash Reconstruction of Pedestrian Accidents Using
Optimization Techniques. Int. J. Impact Eng. 36 (2), 210-219. doi:10.1016/ j.ijimpeng.2008.01.012

Wang, J., Wang, R., Gao, W., Chen, S., and Wang, C. (2020). Numerical Investigation of Impact Injury of a Human Head during Contact Interaction with a Windshield Glazing Considering Mechanical Failure. Int. J. Impact Eng. 141, 103577. doi:10.1016/ j.ijimpeng.2020.103577

Wu, H., Han, Y., Pan, D., Wang, B., Huang, H., Mizuno, K., et al. (2021). The Head AIS 4+ Injury Thresholds for the Elderly Vulnerable Road User Based on Detailed Accident Reconstructions. Front. Bioeng. Biotechnol. 9, 682015. doi:10.3389/fbioe.2021.682015

Yang, J., and Otte, D. (2007). "A Comparison Study on Vehicle Traffic Accident and Injuries of Vulnerable Road Users in China and Germany," in Proceedings of the 20th Experimental Safety Vehicles Conference(Lyon, France.

Yang, J. (2005). Review of Injury Biomechanics in Car-Pedestrian Collisions. Int. J.Veh. Saf. 1 (1-3), 100-117. doi:10.1504/ijvs.2005.007540

Conflict of Interest: Author LS is employed by the company China Automotive Engineering Research Institute Co., Ltd.

The remaining authors declare that the research was conducted in the absence of any commercial or financial relationships that could be construed as a potential conflict of interest.

Publisher's Note: All claims expressed in this article are solely those of the authors and do not necessarily represent those of their affiliated organizations, or those of the publisher, the editors and the reviewers. Any product that may be evaluated in this article, or claim that may be made by its manufacturer, is not guaranteed or endorsed by the publisher.

Copyright $\odot 2021 \mathrm{Li}, \mathrm{Liu}, \mathrm{Li}$, Zhao, Shi, Zhang and Nie. This is an open-access article distributed under the terms of the Creative Commons Attribution License (CC BY). The use, distribution or reproduction in other forums is permitted, provided the original author(s) and the copyright owner(s) are credited and that the original publication in this journal is cited, in accordance with accepted academic practice. No use, distribution or reproduction is permitted which does not comply with these terms. 